\title{
Espíritu emprendedor en los estudiantes de la escuela Panamá de la ciudad de Cuenca-Ecuador
}

\author{
Marcos Eduardo Cantos Ochoa ${ }^{1}$ \\ https://orcid.org/0000-0002-3340-5085 \\ John Edwin López Castillo ${ }^{2}$ \\ Alexandra del Rocío Reyes Cabrera ${ }^{3}$
}

\section{RESUMEN}

Recebido: 05.11.2019

Aceito: 15.12 .2019

Publicado: 13.01 .2020

El objetivo del presente artículo es determinar las competencias emprendedoras, la imagen que tienen sobre los empresarios y las personas que influyen en las decisiones y acciones de los alumnos de noveno y décimo año de la escuela Panamá de la ciudad de Cuenca - Ecuador para ser emprendedores en el futuro. Las bases teóricas se fundamentaron desde la perspectiva de Cordón, et al (1999), Redine (Ed.) (2018), De Jesús (2013), Olarte (2015), Rodríguez y Gómez (2014), Alcaráz (2011), Marulanda, et al (2009), Herrera y Montoya (2013), Bassat (2017), Hernández, et al (2015), Sánchez (2010), Ortíz y Millán (2011), Santamaría, et al (2013) y Ayala (2007). Se recurrió a la investigación descriptiva, el estudio empleó un diseño no experimental y transversal, de campo, la población, estuvo constituida por 79 estudiantes, para el levantamiento de la información, se aplicó un cuestionario de recolección de datos apropiado al objetivo de la investigación. Se concluye que las competencias que prevalecen en los estudiantes se refieren a la "disposición de asumir los riesgos", "trabajo en equipo" que se encuentra dentro de la capacidad de comunicación, visión de futuro en el sentido de la predisposición para "proponer nuevas formas de hacer las cosas", sin embargo, la creatividad no es una fortaleza. La persona emprendedora que más influye en sus acciones y decisiones es su madre, en tanto que definen al empresario como una persona honrada que gana mucho dinero, lo cual en gran porcentaje les motiva para en un futuro tener su propio negocio.

Palabras claves: espíritu emprendedor, competencias emprendedoras, variables del entorno social y de la empresa.

\section{Espirito empreendedor nos alunos da escola Panamá na cidade de Cuenca-Equador \\ RESUMO}

O objetivo do presente artigo é determinar as competências empreendedoras, a imagem que têm sobre os empresários e as pessoas que influenciam as decisões e acções dos alunos do nono e décimo ano da Escola Panamá da cidade de Cuenca - Ecuador para serem empreendedores no futuro. As bases teóricas fundamentaram-se desde a perspectiva de Cordón, et al. (1999), Redine (Ed.) (2018), De Jesús (2013), Olarte (2015), Rodríguez y Gómez (2014), Alcaráz (2011), Marulanda, et al (2009), Herrera e Montoya (2013), Bassat (2017), Hernández, et al. (2015), Sánchez (2010), Ortíz e Millán (2011), Santamaría, et al. (2013) e Ayala (2007). Recurreu-se à investigação descriptiva, utilizando um desenho não experimental e transversal, de campo, a população, esteve constituida por 79 alunos, para a recolha de informação, aplicou-se um questionário contextualizado ao objetivo da investigação. Se conclui que as competências que prevalecem nos alunos referem-se à "disposição de assumir os riscos", "trabalho em grupo" que se encontram dentro da capacidade de comunicação, visão de futuro no sentido da predisposição para "propôr novas formas de fazer as coisas", não obstante, a criatividade não é uma fortaleza. A pessoa empreendedora que mais influi nas suas acções e decisões é sua mãe, uma vez que definem o empresário como uma pessoa honrada que ganha muito dinhero, o aual em grande pecentagem lhes motiva para no futuro ter o seu própio negócio.

Palavras-Chaves: Espírito empreendedor, Competências empreendedoras, Variáveis sociais e Empresa.

\section{Entrepreneurship in the students of the Panama school in the city of Cuenca-Ecuador}

\section{ABSTRACT}

The objective of this article is to determine entrepreneurial competencies, the image they have about entrepreneurs and the people who influence the decisions and actions of the ninth that the ninth and tenth year students of the Panama School in the city of Cuenca- Ecuador have to be entrepreneurs in the future. The theoretical bases were

\footnotetext{
${ }^{1}$ Universidad Catolica de Cuenca, Ecuador. Correo electrónico: mecantoso@ucacue.edu.ec

${ }^{2}$ Universidad Catolica de Cuenca, Ecuador. Correo electrónico: jlopezc@ucacue.edu.ec

${ }^{3}$ Universidad Catolica de Cuenca, Ecuador. Correo electrónico: areves@ucacue.edu.ec
} 
founded from the perspective of Cordón, et al (1999), Redine (Ed.) (2018), De Jesús (2013), Olarte (2015), Rodríguez y Gómez (2014), Alcaráz (2011), Marulanda, et al (2009), Herrera y Montoya (2013), Bassat (2017), Hernández, et al (2015), Sánchez (2010), Ortíz y Millán (2011), Santamaría, et al (2013) and Ayala (2007). Descriptive research was used, the study employs a non-experimental and cross-sectional design, the population of the field is made up of 79 students, for the collection of information, and a data collection questionnaire was applied appropriate to the objective of the research. It is conclude that the competencies that prevail in students refer to the willingness to take risks, teamwork that is within the capacity of communication, vision of the future in the sense of predisposition to propose new ways of doing things. However, creativity is not strength. The entrepreneur who most influences their actions and decisions is their mother, as they define the entrepreneur as an honest person who earns a lot of money, which in large percentage motivates them to have their own business in the future.

Key Words: Entrepreneurial spirit, entrepreneurial skills, variables of the social and business environment.

\section{Introducción}

Emprender es algo que está intrínseco en el ser humano y se entiende como la aptitud para localizar carencias y expectativas insatisfechas, comprometiéndose a un cierto grado de riesgo para poner en marcha proyectos que las satisfagan y así intervenir en el cambio de su propia vida y la de su entorno, requiere esfuerzo, disciplina, talento, formación y, sobre todo, mucha pasión y dedicación. El estudio del emprendimiento de acuerdo a Toapanta, et al (2018) ha cobrado importancia en el ámbito educativo y estatal dado a su impacto positivo en el desarrollo económico y social de los países, por lo que el desconocimiento del perfil emprendedor del estudiante en los centros educativos ocasiona que los recursos destinados a su fortalecimiento no se asignen eficientemente. Para estudiarlo debemos reconocer su complejidad como punto de partida, en su análisis es necesario identificar las conductas, motivación y competencias que tienen las personas relacionadas con la sensibilidad de emprender. Al respecto Santos, Caetano y Curral (2013, citado por Rodríguez y Gómez 2014), aseveran que cada persona posee un potencial para el emprendimiento, el mismo que está constituido por competencias psicológicas, sociales y gerenciales, así como motivaciones emprendedoras.

En la definición que se da al espíritu emprendedor como competencia, Sobrado y Fernández (2010, p.16) lo entienden como "la habilidad de la persona para transformar las ideas en actos. Está relacionado con la creatividad, la innovación y la asunción de riesgos, así como con la habilidad para planificar y gestionar proyectos con el fin de alcanzar objetivos".

Según lo refieren Carlos, et al (2015, p.46) "es relevante estudiar al espíritu emprendedor, que se entiende como las características de la personalidad que se manifiestan en la forma de pensar y actuar vinculadas con la búsqueda y el aprovechamiento de las oportunidades".

En el ámbito educativo, hacer referencia al espíritu emprendedor implica aludir dos tipos de competencias, unas genéricas y válidas para el colectivo estudiantil y el conglomerado social y otras particulares, ligadas al:

"desarrollo profesional como empresario/a y que son patrocinadas por las primeras. Entre los objetivos de la educación "tiene que estar siempre presente el fomento de las cualidades personales que constituyen la base del espíritu emprendedor, a saber: la creatividad, la iniciativa, la responsabilidad, la capacidad de afrontar riesgos y la independencia o autonomía en el trabajo" (Toapanta et al, 2018, p.76).

En este escenario, comparece el estudiante a nivel de Educación General Básica (EGB), que, con esa energía e ilusión propia de su edad, se hace presente con sus ideas y anhelos de crear o generar bienes y servicios que sirvan para dar posibles soluciones a los problemas de los seres humanos y de la sociedad. Cada estudiante básicamente posee sus propias competencias, capacidades y preferencias profesionales, unas innatas y otras que son el fruto de la inspiración en padres, familiares, docentes, empresarios y amigos.

En este contexto es pertinente distinguir las competencias emprendedoras que tienen los estudiantes de noveno y décimo año de Educación General Básica de la escuela Panamá de la ciudad de Cuenca - Ecuador, con el fin fomentar entre el alumnado aptitudes que permitan el desarrollo del espíritu emprendedor desde edades tempranas. Por lo que el objetivo del presente 
artículo es determinar las competencias emprendedoras, la imagen que tienen sobre los empresarios y las personas que influyen en las decisiones y acciones de los alumnos de noveno y décimo año de la escuela Panamá de la ciudad de Cuenca - Ecuador para ser emprendedores en el futuro.

En el presente artículo, en primer lugar, se detalla la metodología a seguir para el análisis de las competencias emprendedoras y factores sociales y de la empresa de los alumnos de Educación General Básica de la escuela Panamá; a continuación, se aborda la exposición de las principales bases teóricas de las variables de competencias emprendedoras, así como los factores sociales y de la empresa que influyen en su iniciativa de emprender; posteriormente se presentan los resultados $y$, finalmente, se emiten las conclusiones.

\section{Materiales y métodos}

Para conocer el perfil emprendedor se hizo referencia a las competencias emprendedoras de autoconocimiento, visión de futuro, energía, planificación comunicación, precepción de riegos, creatividad y personalidad proactiva, mientras que para determinar los factores sociales y de la empresa que influyen en la intensión de emprender se hizo alusión al aprendizaje social, imagen del empresario y deseabilidad desde la perspectiva de Cordón, et al (1999), Redine (Ed.) (2018), De Jesús (2013), Olarte (2015), Rodríguez y Gómez (2014), Alcaráz (2011), Marulanda, et al (2009), Herrera y Montoya (2013), Bassat (2017), Hernández, et al (2015), Sánchez (2010), Ortíz y Millán (2011), Santamaría, et al (2013) y Ayala (2007), entre otros.

En cuanto a la metodología empleada para llevar a cabo este estudio, es fundamentalmente descriptiva, porque pretendió determinar la condición de las competencias emprendedoras en el ámbito de la Educación General Básica, tal y como se presentaron en la realidad. Asimismo, en la investigación se empleó un diseño no experimental y transversal, de campo, debido a que las variables relacionadas con las competencias emprendedoras no fueron variadas intencionalmente y la recolección de datos se realizó en una sola observación. La población, estuvo conformada por 79 estudiantes de la escuela Panamá de la ciudad de Cuenca, Ecuador, representada por el alumnado del noveno y décimo año de dicha institución, en consideración de que su plan de estudios incluye la materia de emprendimiento. Para el levantamiento de la información, se empleó un cuestionario pertinente con el objetivo de la investigación, en este sentido para la recopilación de los datos relacionados con las variables de competencias emprendedoras se utilizó un baremo cuali-cuantitativo conforme se explica en el cuadro 1 y los resultados se demuestran a través de una media aritmética de respuestas. En relación a los factores sociales y de la empresa que inciden en la iniciativa de emprender los resultados se demuestran en porcentaje; el análisis de los datos se realizó bajo la estadística descriptiva.

Cuadro No.1

Baremo cuali-cuantitativo de la Escala de Likert

\begin{tabular}{|c|c|}
\hline Valor Cuantitativo & Valor Cualitativo \\
\hline 5 & Siempre \\
\hline 4 & Casi siempre \\
\hline 3 & Ocasionalmente \\
\hline 2 & Casi nunca \\
\hline 1 & Nunca \\
\hline
\end{tabular}

Fuente: Elaboración Propia.

\section{Variables de competencias emprendedoras}

Las competencias emprendedoras de acuerdo a Martínez y Carmona (2009) y Rodríguez y Gómez (2014) hacen referencia a características y rasgos de personalidad individuales que implican capacidades, destrezas, habilidades y aptitudes que puestas en práctica permiten resolver con éxito las diferentes problemáticas y hacen posible que el emprendedor se desempeñe de manera idónea en el proceso de emprender. 
A la hora de analizar el espíritu emprendedor de los estudiantes que están cursando los estudios de secundaria, es importante destacar las siguientes competencias:

\section{Autoconocimiento}

Es fundamental realizar un reconocimiento de nuestras capacidades, creencias, temores, debilidades, metas y el nivel de satisfacción en nuestras relaciones interpersonales, al respecto Whetten y Cameron (2004, p.54) indican que "el autoconocimiento ha sido identificado como un aspecto crucial de la inteligencia emocional y es más poderoso para pronosticar el éxito en la vida que el coeficiente intelectual".

El autoconocimiento y reconocimiento de manera crítica y objetiva de, lo que somos, lo que creemos, lo que valoramos, nuestras capacidades, metas y temores, etc., permitirá tener una visión sólida para afrontar adecuadamente los problemas y desafíos en cualquier situación y lograr una satisfacción integral.

Cordón, et al (1999) y Redine (Ed.) (2018) coinciden en que el autoconocimiento en el emprendimiento se basa en que el sujeto entienda sus ventajas, pericia, destrezas, instintos y recursos, en una diversidad de aspectos; como, por ejemplo, su equilibrio emocional, sus capacidades interpersonales, su autoestima y su disposición hacia el trabajo, para utilizarlos, en base a la experiencia y alcanzar objetivos. Esta forma de entender el autoconocimiento resulta medular para desarrollar su sensibilidad por la realidad del entorno en el cual se desenvuelve, así como habilidades interpersonales.

El autoconocimiento de los estudiantes de noveno y décimo año de la escuela Panamá se evaluó a partir de los siguientes ítems: uso mis habilidades; tengo iniciativa para hacer las cosas; tengo en cuenta posibles riesgos; asumo mi parte de responsabilidad.

\section{Visión de futuro}

La visión de futuro es una competencia que permite advertir tendencias y anticipar lo que va a ocurrir, De Jesús (2013, p. 31) señala que es una "habilidad para ver más allá de la realidad presente, crear e inventar aquello que no existe y convertirlo en algo concreto y tangible que apasiona y es fuente primaria de inspiración para las acciones que mueven al ser humano".

La visión de futuro, es un método para fijar metas y delinear el camino para alcanzarlas, nos incita a actuar de forma proactiva proyectando el mañana, desde nuestras actitudes y fortalezas, nos alienta y nos da la fuerza de voluntad suficiente para originar o generar los cambios para su consecución, en este ámbito los ítems analizados hacen referencia a: propongo nuevas formas de hacer las cosas; tengo en cuenta posibles resultados y doy soluciones.

\section{Energía}

El Emprendedor de acuerdo a Olarte (2015) se desenvuelve en un universo dinámico, donde todos los seres vivos son fuente de energía y no sólo la genera, sino que la encauza, estimulando procesos y situaciones en su contexto. La energía es primordial para emprender, pues de ella depende la voluntad para ordenarse, arremeter los desafíos y decisiones del día a día. Cada pequeña victoria retroalimenta él fervor y las energías para proseguir. "Por lo tanto, el emprendedor modelo tiene una gran necesidad de realización personal. Es una persona con ilusiones, lleno de energía y creatividad; tiene confianza en sí mismo; se compromete entera y totalmente” (Ayala, 2007, p. 1156).

La energía de los alumnos se apreció a partir de los siguientes ítems: busco superarme; mantengo el ánimo; cumplo lo prometido y me exijo en hacer bien las cosas.

\section{Planificación}

La planificación es un factor clave para tener éxito en el ámbito donde se haya decidido emprender. $\mathrm{Al}$ respecto Mavila, et al $(2009$, p. 33) se refiere a la planificación como: 
"la capacidad de pensar antes de actuar, identificando metas concretas o resultados que se desean alcanzar y diseñar planes consistentes para desempeñarse de manera que se aproveche las oportunidades y anular o disminuir las amenazas del entorno. Comprende:

A. El establecimiento de metas.

B. La búsqueda de información, y

C. La planificación sistemática y el control".

En función de lo expuesto, para medir la competencia de planificación en los estudiantes se consideraron los siguientes ítems: utilizo poco a poco mis recursos; evalúo lo que hago en cada momento y me fijo metas.

\section{Comunicación}

La comunicación es la base del emprendimiento, esta destreza hace posible que el emprendedor ejerza adecuadamente el proceso de emprender, según Rodríguez y Gómez (2014) la comunicación enfoca el trabajo a alcanzar objetivos teniendo en cuenta la relación costo- beneficio de las acciones, influye en otros para llegar a acuerdos, solucionar conflictos y realizar alianzas donde las partes resultan beneficiadas.

La comunicación no solamente permite solventar las pugnas también ayuda a una relación adecuada entre los miembros del equipo promoviendo una mayor productividad, para evaluar esta competencia en los alumnos se utilizaron los siguientes ítems: escucho y atiendo al otro; trabajo en equipo; influyo en los demás y busco nuevas amistades.

\section{Percepción de riesgos}

De acuerdo a Alcaráz (2011, p.8) el riesgo "es un elemento que, para algunos autores constituye una característica fundamental del acto de emprender, pues consideran que el emprendedor es un "tomador de riesgos" que pondera los beneficios y asume los peligros que son inherentes a su consecución".

Marulanda, et al (2009) y Herrera y Montoya (2013) desde la visión cognitiva, basada en los comportamientos, pensamientos o representaciones del individuo coinciden en que el emprendedor es un individuo que presenta necesidades de cumplimiento y dependencia, así como como un gusto por el riesgo.

Ser emprendedor implica correr riesgos, la tolerancia y actitudes positivas hacia el mismo predicen el éxito, aunque la probabilidad de triunfar sea baja o no esté definida es necesario la toma de decisiones bajo condiciones de incertidumbre, pues si no corre el riesgo, no se puede ganar. El emprendedor acepta el riesgo inherente en la toma de decisiones y persevera en la puesta en marcha de su proyecto, en este sentido se preguntó a los estudiantes si están dispuestos a asumir dichos riesgos.

\section{Creatividad}

La creatividad es una actitud que trata de transformar la realidad, se la considera como un eje básico de supervivencia necesaria para resolver problemas cotidianos que se presentan en la vida social y laboral. Bassat (2017) refuerza este enunciado al manifestar que la creatividad es el conjunto de aptitudes vinculadas a la personalidad de los individuos, que le permiten a partir de una indagación precedente y mediante una serie de procesos cognitivos, transformar la información y dar solución a los problemas con originalidad y eficacia.

En general, según Hernández, et al (2015) la creatividad es una actitud mental y una técnica de pensamiento que tiene el ser humano para producir nuevas ideas, enfoques y acciones, engloba facultades, fuerzas y el intelecto que se revelan en innovadoras formas para examinar la naturaleza de un dilema, la creatividad es resolutiva y permite producir una serie de alternativas de solución. 
La creatividad de los estudiantes se ha evaluado a partir de los siguientes ítems: frecuentemente tengo ideas originales y los pongo en práctica; no me resulta difícil encontrar soluciones a un mismo problema; disfruto buscando nuevas formas de ver las cosas y me entusiasma realizar cosas nuevas e inusuales.

\section{Personalidad proactiva}

Las personas proactivas de acuerdo a Sánchez (2010) reconocen las oportunidades y proceden sobre ellas, exponen ideas, desarrollan acciones directas y persisten hasta lograr un cambio representativo, ser proactivo implica anticipar y eludir inconvenientes antes de que acontezcan y una disposición a la acción que implica una apreciación de los preceptos, la entereza y un alto nivel de persistencia para ejecutar dicho cambio.

La proactividad es una capacidad imprescindible en la llamada orientación emprendedora que supone a más de la perseverancia, la aptitud para responsabilizarse ante el fracaso. La voluntad de emprender está positivamente adjunta a una personalidad proactiva. Para reunir información sobre los rasgos de personalidad proactiva de los estudiantes se aplicaron los siguientes ítems: si veo algo que no me gusta puedo cambiarlo; si creo en algo lo haré, sin importarme las probabilidades de éxito, me gusta defender mis ideas y siempre estoy buscando mejores formas de hacer las cosas.

\section{Factores sociales y de la empresa}

Una vez definidas las competencias que se relacionan con la iniciativa de emprender de los estudiantes de educación general básica, Ortíz y Millán (2011, p.219) manifiestan que es esencial "añadir a las características personales que tradicionalmente han explicado la iniciativa empresarial de los individuos, las variables del entorno social y de la empresa. Todas ellas componen el escenario en el que se lleva a cabo la iniciativa emprendedora". En este ámbito abordamos el aprendizaje social, la imagen del empresario y la deseabilidad.

\section{Aprendizaje social}

Ortíz y Millán (2011) entienden que existen ciertos aspectos sociales que demuestran la predisposición a emprender, y que estimulan una conducta emprendedora. Es decir, tenemos la convicción de que dicha conducta es el producto de factores personales y sociales que se enlazan directamente con un determinado perfil emprendedor.

Comparte esta misma idea Santamaría, et al (2013) y por ello afirma, que, hay ciertos aspectos del ámbito social, como los precedentes familiares, la costumbre o el aprendizaje previo, el sistema de valores, entre otros, que impulsan o impiden el surgimiento de emprendedores

"la idea fundamental de esta teoría, lo que la identifica y la diferencia de otros modelos, es el protagonismo que le confiere a la presión que ejerce el entorno familiar, educativo, laboral, en definitiva, social, en el surgimiento de la conducta emprendedora. En otras palabras, los grupos de referencia y/o de pertenencia actúan como modelos, como factores importantes que explican la intención emprendedora a partir de la interacción entre aspectos personales y sociales" (Ortíz y Millán, 2011, p.223).

Para definir los factores sociales y personales que influyen en la iniciativa de emprender de los estudiantes, se les pregunto sobre la persona que influye en sus acciones y decisiones y sí la misma es emprendedora.

\section{Imagen del empresario}

Tal y como hoy lo conocemos, el empresario es el principal responsable del desarrollo económico, su figura hace hincapié en el papel de impulsor de la economía, esta imagen es la que refleja la capacidad y motivación que tienen los individuos en su intensión, de emprender. 
Para establecer su imagen frente a los alumnos se utilizaron los siguientes ítems: persona honrada; persona dinámica; persona que labora con los trabajadores y persona que gana mucho dinero.

\section{Deseabilidad}

De acuerdo a Ayala (2007) el ámbito cultural y social, las costumbres, los valores y conductas de los integrantes de una comunidad condicionan las determinaciones que conducen a ciertas prácticas. En el caso de la función empresarial, las percepciones de la deseabilidad y viabilidad son el fruto del contexto sociocultural que deciden qué prácticas o procederes serán tomados en cuenta y en el futuro adoptados.

Para medir la deseabilidad de emprender en el futuro de los estudiantes de educación general básica, se les pregunto si les gustaría: tener un negocio propio, trabajar en una empresa o ser empleado público.

A continuación, el siguiente cuadro resume tanto las competencias emprendedoras como las variables del entorno social y de la empresa que caracterizan e influyen en la intensión de emprender de los estudiantes de noveno y décimo de la escuela Panamá de la ciudad de Cuenca Ecuador.

\section{Cuadro No.2}

Competencias emprendedoras y variables del entorno social y de la empresa

\begin{tabular}{|c|l|}
\hline \multirow{5}{*}{ Competencias emprendedoras } & Autoconocimiento \\
\cline { 2 - 2 } & Visión de futuro \\
\cline { 2 - 2 } & Energía \\
\cline { 2 - 2 } & Planificación \\
\cline { 2 - 2 } & Comunicación \\
\cline { 2 - 2 } & Percepción de riesgos \\
\cline { 2 - 2 } & Creatividad \\
\cline { 2 - 2 } $\begin{array}{c}\text { Variables del entorno social y de la } \\
\text { empresa }\end{array}$ & Personalidad proactiva \\
\cline { 2 - 2 } & Imagendizaje social del empresario \\
\cline { 2 - 2 } & Deseabilidad \\
\hline
\end{tabular}

Fuente: Elaboración Propia.

\section{Resultados de investigación}

Entre las competencias emprendedoras se abordó las aptitudes relacionadas con el autoconocimiento, visión de futuro, energía, planificación comunicación, precepción de riegos, creatividad y personalidad proactiva, mediante un cuestionario que recoge la percepción de los estudiantes de acuerdo a los ítems de competencia conforme se reflejan en el cuadro 3., empleando una escala likert de 1: nunca a 5 siempre.

Cuadro No. 3

Variables de competencias emprendedoras

\begin{tabular}{|c|l|}
\hline Competencias emprendedoras & \multicolumn{1}{|c|}{ Ítems de competencia } \\
\hline \multirow{4}{*}{ Autoconocimiento } & Uso mis habilidades \\
\cline { 2 - 2 } & Tengo iniciativa para hacer las cosas \\
\cline { 2 - 2 } & Tengo en cuenta posibles riesgos \\
\cline { 2 - 2 } & Asumo mi parte de responsabilidad \\
\hline \multirow{4}{*}{ Visión de futuro } & Propongo nuevas formas de hacer las cosas \\
\cline { 2 - 2 } & Tengo en cuenta posibles resultados \\
\cline { 2 - 2 } & Doy soluciones \\
\hline \multirow{3}{*}{ Energía } & Busco superarme \\
\cline { 2 - 2 } & Mantengo el ánimo \\
\cline { 2 - 2 } & Cumplo lo prometido \\
\hline
\end{tabular}




\begin{tabular}{|c|c|}
\hline & Me exijo en hacer bien las cosas \\
\hline \multirow{3}{*}{ Planificación } & Utilizo poco a poco mis recursos \\
\hline & Evalúo lo que hago en cada momento \\
\hline & Me fijo metas \\
\hline \multirow{4}{*}{ Comunicación } & Escucho y atiendo al otro \\
\hline & Trabajo en equipo \\
\hline & Influyo en los demás \\
\hline & Busco nuevas amistades \\
\hline Percepción de riesgos & Esta dispuesto a asumir los riesgos \\
\hline \multirow{4}{*}{ Creatividad } & $\begin{array}{l}\text { Frecuentemente tengo ideas originales y los pongo en } \\
\text { práctica }\end{array}$ \\
\hline & $\begin{array}{l}\text { No me resulta difícil encontrar soluciones a un mismo } \\
\text { problema }\end{array}$ \\
\hline & Disfruto buscando nuevas formas de ver las cosas \\
\hline & Me entusiasma realizar cosas nuevas e inusuales \\
\hline \multirow{4}{*}{ Personalidad proactiva } & Si veo algo que no me gusta puedo cambiarlo \\
\hline & $\begin{array}{l}\text { Si creo en algo lo haré, sim importarme las } \\
\text { probabilidades de éxito }\end{array}$ \\
\hline & Me gusta defender mis ideas \\
\hline & $\begin{array}{l}\text { Siempre estoy buscando mejores formas de hacer las } \\
\text { cosas }\end{array}$ \\
\hline
\end{tabular}

Fuente: Elaboración Propia.

Para el análisis de los resultados obtenidos se procedió a realizar una comparación con la información obtenida por García y Moreno (2012) en su estudio denominado "Aptitudes emprendedoras de los alumnos de Educación Secundaria Obligatoria y Bachillerato en la Región de Murcia".

De acuerdo al comportamiento de las respuestas la competencia mejor valorada por los estudiantes de la escuela Panamá se refiere a la percepción de riesgos (4,60 - siempre), mientras que para el alumnado de la región de Murcia prevalece la competencia de energía (3,87- casi siempre), seguido por la comunicación en ambos casos con 3,75 y 3,77 (casi siempre) respectivamente, así mismo hacemos hincapié en la creatividad como la aptitud menos valorada en los dos estudios 1,80 (casi nunca) y 3,40 (ocasionalmente) de acuerdo a lo datos reflejados en el gráfico 1.

\section{Gráfico No. 1}

Ranking de competencias emprendedoras

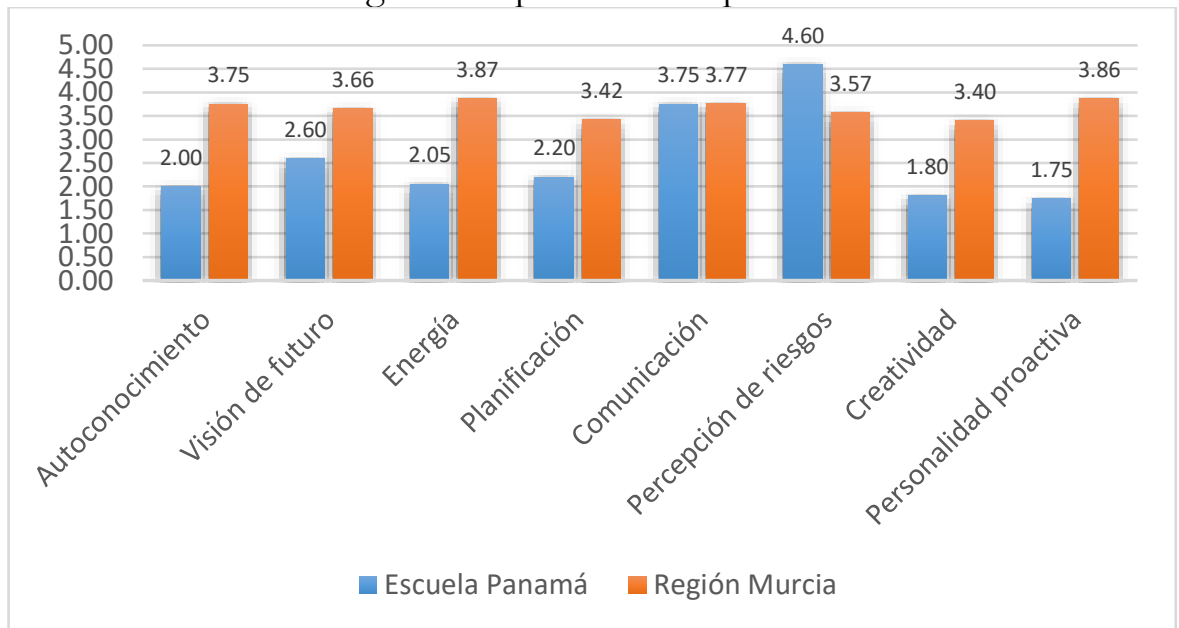

Fuente: Escuela Panamá (2018) y García y Moreno (2012) 
En relación a los ítems de competencia los alumnos de la región de Murcia "puntúan con mayores valores su capacidad para asumir riesgos "Creo en el dicho: Quien no arriesga no gana" (4,12 - casi siempre), y "Creo que en la vida hay que tomar riesgos para ganar más o alcanzar metas más altas" (4,08 - casi siempre) así como actitudes proactivas como "Me gusta defender mis ideas, incluso en contra de la oposición de los demás". (3.91 - casi siempre). Por otra parte, los ítems menos valorados son la capacidad para evaluar lo que hacen en cada momento (3,05 - ocasionalmente), "No me resulta difícil encontrar varias soluciones a un mismo problema". (2.85 - ocasionalmente), y "No me asusta la idea de lo desconocido". (2,67 - ocasionalmente)" (García y Moreno, 2012, p.20).

En este ámbito los estudiantes de la escuela Panamá concuerdan con los de la región de Murcia y otorgan una mayor calificación a su capacidad de "Está dispuesto a asumir los riesgos (4,62 siempre). Después de este indicador dentro de los ítems que los estudiantes puntúan con mayores valores tenemos: "trabajo en equipo" (3,73 - casi siempre) correspondiente a la competencia de comunicación, "propongo nuevas formas de hacer las cosas" (2,59 - ocasionalmente) dentro de la competencia visión de futuro, en la actitud de planificación "utilizan poco a poco sus recursos" (2,22 - casi nunca), y el (2,03 - casi nunca) "buscan superarse" (energía).

Entre los ítems menos valorados que se corresponden con el estudio realizado en los estudiantes de la región de Murcia se encuentran "evaluó lo que hago en cada momento" (1,33 - nunca) y "no me resulta difícil encontrar varias soluciones a un mismo problema". (2,85 - ocasionalmente).

Por otra parte, los ítems de competencia menos puntuados por los estudiantes de la escuela Panamá son: "escucho y atiendo al otro" (0,63 - nunca) de comunicación "mantengo el ánimo" ( 0,60 - nunca) de energía y "Si creo en algo lo haré, sin importarme las probabilidades de éxito" de personalidad proactiva y "tengo en cuenta posibles riesgos" (0,54 - nunca) de autoconocimiento. En el gráfico 2., se demuestran por orden de respuesta los ítems de competencia.

Gráfico No. 2

Ítems competencias emprendedoras

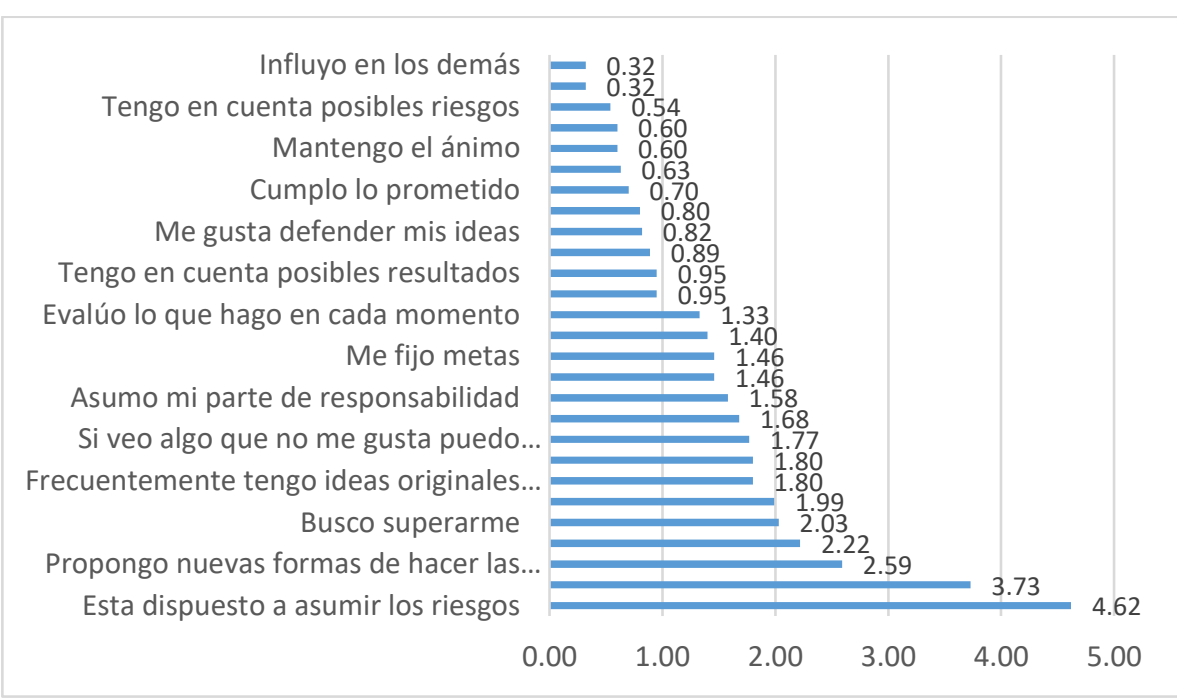

Fuente: Elaboración Propia.

En cuanto a las variables del entorno social y de la empresa, al referirnos al aprendizaje social se consultó a los estudiantes quién es la persona que influye más en sus decisiones y acciones y si la misma es emprendedora, "en concreto de acuerdo a García y Moreno (2012) el 46.10\% de los estudiantes de la región de Murcia eligen a la madre como su ejemplo a seguir. Esta apreciación coincide con el estudio realizado en la escuela Panamá, pues el 53\% de los estudiantes destacaron 
que su madre es la persona más influyente, mientras que el $28 \%$ en el caso de los dos estudios elijen la figura del padre. Los resultados muestran que las personas elegidas como referentes por los estudiantes sí son emprendedoras (25\% y $63 \%$ respectivamente).

Gráfico No. 3

Aprendizaje Social



Fuente: Elaboración Propia.

Sobre la imagen del empresario, la percepción de los estudiantes de la escuela Panamá y los de la región de Murcia presenta diferencias significativas en los ítems persona honrada (33\% - 22,09\%) y persona dinámica $(19 \%-25,70 \%)$ respectivamente. Concordando en los ítems que se refieren al empresario como persona que labora con los trabajadores $(23 \%-24,67 \%)$ y persona que gana mucho dinero $(25 \%-27,54 \%)$.

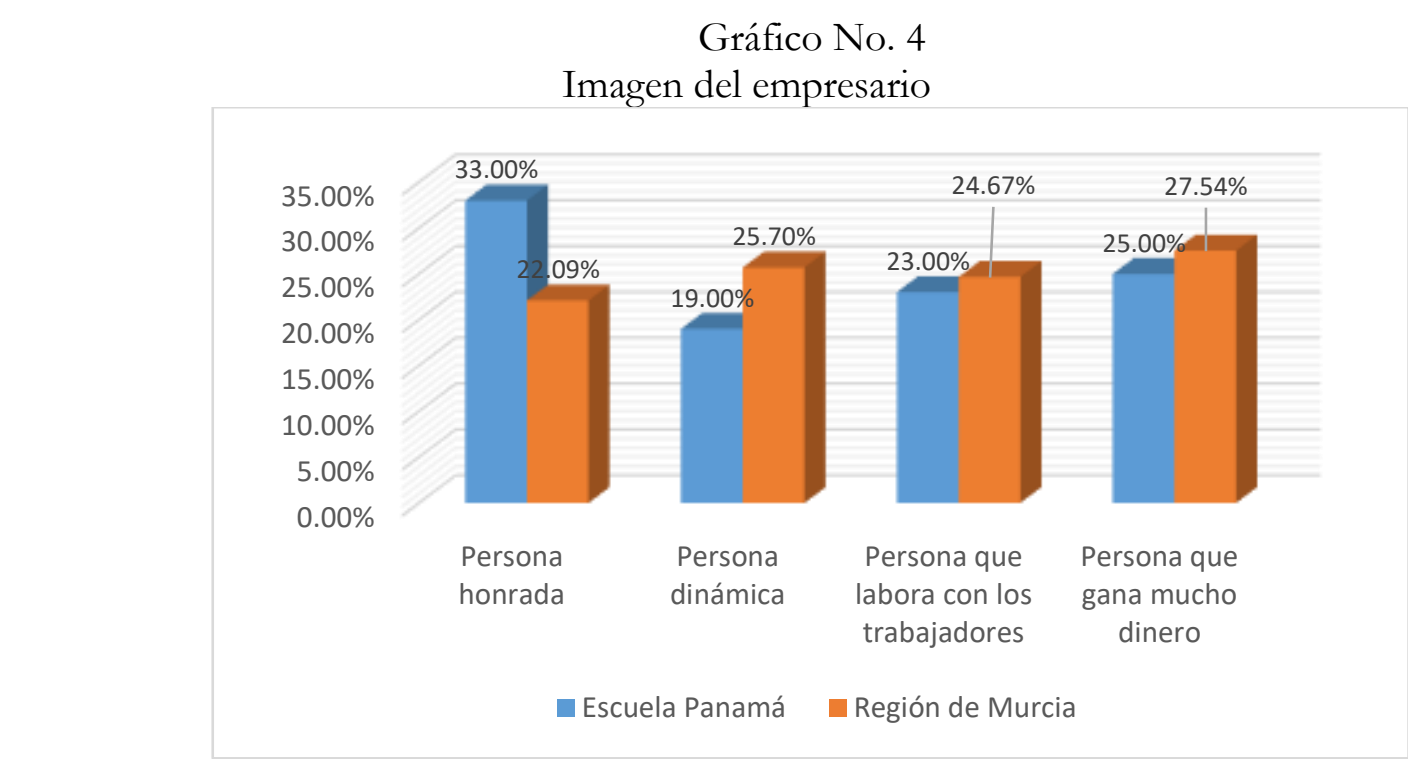

Fuente: Elaboración Propia. 
Referente a su deseabilidad, el gráfico 5., demuestra que: tanto a los estudiantes de la escuela Panamá, (65\%), como a los estudiantes de la región de Murcia (72,20\%) les gustaría en el futuro tener un negocio propio.

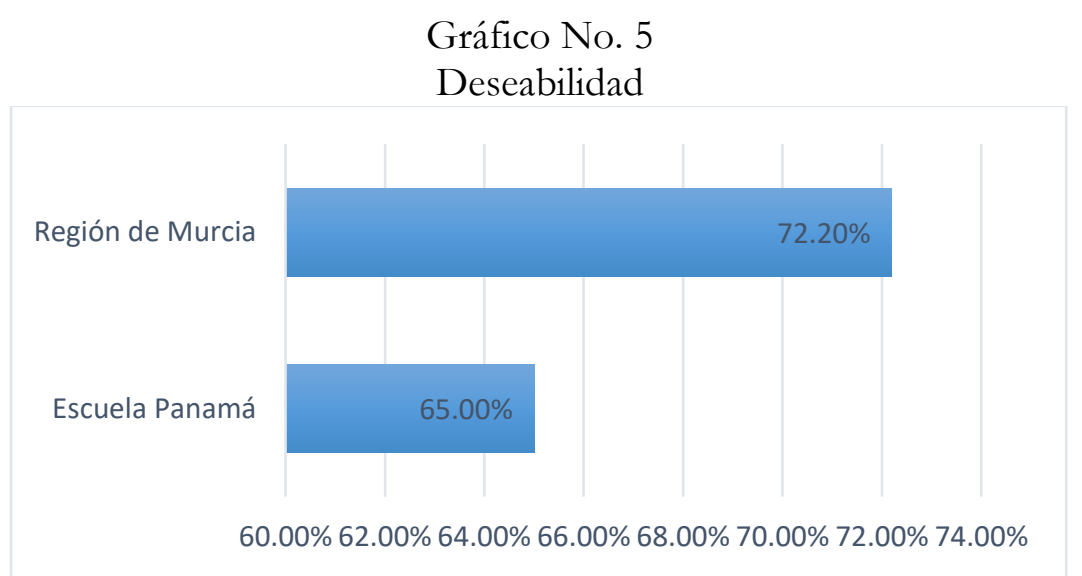

Fuente: Elaboración Propia.

\section{Conclusiones}

Dentro de las actitudes fundamentales para la actividad emprendedora, la persona debe ser autónoma, creativa, tener visión de futuro, dispuesto a asumir riesgos, habilidad para comunicarse, planificar, ser decidido, flexible, persistente, tener iniciativa, tolerar la ambigüedad, tener motivación al logro, autoconfianza y control interno. En este sentido la tendencia centrada en el análisis de las competencias emprendedoras desde el punto de vista de las peculiaridades y personalidad de los estudiantes indican que:

La competencia que prevalece en mayor grado en los alumnos del noveno y décimo año de la escuela Panamá de la ciudad de Cuenca - Ecuador, se refiere a la "percepción de riesgos", lo cual es un aspecto muy favorable, pues se sabe que, ser emprendedor implica la disposición de asumir los riesgos, la tolerancia y actitudes positivas hacia el mismo predicen el éxito aunque la probabilidad de triunfar sea baja o no esté definida, es necesario, la toma de decisiones bajo condiciones de incertidumbre pues, si no se corre el riesgo no se puede ganar.

Así mismo, siendo la comunicación la base del emprendimiento, esta capacidad, también se destaca en los alumnos. Este resultado al momento de emprender traerá muchos beneficios ya que influye en los demás para llegar a acuerdos, solucionar conflictos y realizar alianzas donde las partes resultan beneficiadas. Esta destreza hace posible que desempeñen adecuadamente el proceso de emprender, ayuda a una relación apropiada entre los miembros del equipo promoviendo una mayor productividad.

La visión de futuro es una competencia emprendedora necesaria para alcanzar los sueños personales, familiares, educativos y por supuesto empresariales. Al respecto, más de la mitad de los estudiantes de la escuela Panamá tienen una predisposición para "proponer nuevas formas de hacer las cosas". Este punto de referencia de éxito, permitirá cambiar los paradigmas y ser más competitivos para superar los desafíos, concede la fuerza de voluntad suficiente para originar o generar los cambios para su consecución.

También dentro de las aptitudes que caracterizan el espíritu emprendedor de los estudiantes, esta, la capacidad de planificar, lo cual es un factor clave para tener éxito en el ámbito donde se decidan 
emprender. Esta destreza permite pensar antes de proceder, determinando objetivos precisos o resultados que se desean lograr y trazar planes congruentes para desempeñarse de manera óptima aprovechando las oportunidades y revocando o menguando las amenazas del entorno.

Al hablar de la creatividad, debemos indicar que la misma no es una fortaleza en los estudiantes encuestados pues, el ítem "frecuentemente tengo ideas originales y los pongo en práctica" no se sido puntuado adecuadamente por los mismos. En este ámbito es necesario dedicar tiempo a desarrollar el pensamiento creativo y potenciar la curiosidad, esto conducirá al estudiante a informarse y a pensar por sí mismo, generando una dinámica positiva que impulsará, en última instancia, la creatividad.

Al referirnos a las variables del entorno social y de la empresa podemos manifestar que: el aprendizaje social se vincula directamente con el perfil emprendedor de la mayoría del alumnado de la escuela Panamá a través de factores personales y sociales que estimulan esta inclinación, en este sentido la observación de su madre en calidad de emprendedora, es el modelo de referencia que les transmite actitudes, valores y comportamientos que influyen en sus acciones y decisiones de emprender.

$\mathrm{Al}$ resaltar la imagen del empresario, lo estudiantes lo definen como una persona honrada que gana mucho dinero, esta apreciación si bien es la motivación en su intención de emprender, es necesario cambiar su valoración social y no mirarlo como una persona que, insensible a los problemas de la colectividad desarrolla su actividad pensando únicamente en su beneficio. Es necesario que se entienda bien su rol en la sociedad como generador de riqueza, empleo y bienestar, en este ámbito el sistema educativo es el indicado a mejorar la cultura empresarial para generar emprendedores que impulsen el crecimiento y desarrollo económico del país.

Finalmente, En el caso de la deseabilidad el entorno social y cultural, los valores, actitudes y hábitos de los miembros de la sociedad en la cual se desenvuelven los estudiantes de la escuela Panamá, determinan un modelo emprendedor positivo que repercute en su decisión de emprender y se asocian con la posibilidad de tener su negocio propio en un futuro.

\section{Referencias Bibliográficas}

Alcaráz, Rafael. (2011). El emprendedor de éxito (cuarta edición). Editorial McGrawHill. México.

Ayala, Juan. (2007). Conocimiento, innovación y emprendedores: camino al futuro. Editorial Universidad de la Rioja. España.

Bassat, Luís. (2017). La creatividad (primera edición). Editorial Conecta. España.

Carlos, Carmen; Contreras, Luís; Silva, María y Liquidano, María. (2015). El espíritu emprendedor y un factor que influencia su desarrollo temprano. Revista Conciencia Tecnológica. $\mathrm{N}^{\circ}$ 49. México (Pp.46-51). Disponible http:/ / www.redalyc.org/articulo.oa?id=94438997006 consulta: 10/10/2019.

Cordón, Eulogío; Rubio, Enrique y Agote, Ángel. (1999). Actitudes hacia la creación de empresas: un modelo explicativo. Revista Europea de Dirección y Economía de la Empresa. Vol.8, N³. España (Pp.37-52).

De Jesús, Ángel. (2013). 5 estrategias para lograr su visión de futuro: Como utilizar sus talentos para obtener resultados extraordinarios en su vida (primera edición). Editorial Palibrio. Estados Unidos de América.

García, Domingo y Moreno, Inmaculada. (2012). Aptitudes emprendedoras de los alumnos de Educación Secundaria Obligatoria y Bachillerato en la Región de Murcia (primera edición). Editorial Universidad Politécnica de Cartagena, Servicio de Documentación. España.

Hernández, Isabel; Alvarado, Juan y Luna, Sara. (2015). Creatividad e innovación: competencias genéricas o transversales en la formación profesional. Revista Virtual Universidad Católica 
del Norte. No44. Colombia (Pp.135-151). Recuperado de https://www.redalyc.org/pdf/1942/194238608010.pdf Consulta: 10/11/2019.

Herrera, Carmen y Montoya, Luz. (2013). El emprendedor: una aproximación a su definición y caracterización. Revista Punto de vista. Vol.4, N 7. Colombia (Pp.7-30). Disponible en https://dialnet.unirioja.es/servlet/articulo? codigo=4776922 consulta: 10/10/2019

Martínez, Francisco y Carmona, Gabriel. (2009). Aproximación al concepto de "competencias emprendedoras": valor social e implicaciones educativas. Revista Iberoamericana sobre Calidad, Eficacia y Cambio en Educación. Vol.7, N 3. España (Pp.153-168). Disponible en http://www.rinace.net/reice/numeros/arts/vol7num3/art6.pdf consulta: 10/10/2019

Marulanda, Jorge; Correa, Geovanny y Mejía, Luís. (2009). Emprendimiento: visiones desde las teorías del comportamiento humano. Revista Escuela de Administración de Negocios. $\quad \mathrm{N}^{\circ}$ 66. Colombia (Pp.153-168). Disponible en: http://www.redalyc.org/articulo.oa?id=20620269008 consulta: 10/10/2019

Mavila, Daniel; Tinoco, Oscar y Campos, César. (2009). Factores influyentes en la capacidad emprendedora de los alumnos de la Universidad Nacional Mayor de San Marcos. Revista Industrial Data. Vol.12, $\mathrm{N}^{\circ}$ 2. Perú (Pp.32-39). Disponible en: https://www.researchgate.net/publication/307180587 Factores influyentes en la capa cidad emprendedora de los alumnos de la Universidad Nacional Mayor de San M arcos consulta: 10/10/2019

Olarte, María (2015). Emprendimiento: una fuente inagotable de energía. Universidad de Salamanca. Extraído de https://catedraemprendedores.org/index.php/blog-ceusal/58emprendimiento-una-fuente-inagotable-de-energia Consulta: 05/09/2019.

Ortíz, Pilar y Millán, Ana. (2011). Emprendedores y empresas: la construcción social del emprendedor. Revista Colección Lan Harremanak. Vol.24, N 1. España (Pp.219-236). Disponible

en https://www.ehu.eus/ojs/index.php/Lan Harremanak/article/view/4695/4481 consulta: 10/10/2019

REDINE (Ed.) (2018). Conference Proceedings EDUNOVATIC 2017: 2nd Virtual International Conference on Education, Innovation and ICT (primera edición). Editorial Adaya Press. Países Bajos.

Rodríguez, Diana y Gómez, Adriana. (2014). Las competencias emprendedoras en el departamento de Boyacá. Revista Apuntes del CENES. Vol. 33, N 58. Colombia (Pp.217-242). Disponible en: http://www.redalyc.org/articulo.oa?id=479547210009. Consulta: $13 / 11 / 2019$.

Sánchez, José. (2010). Evaluación de la personalidad emprendedora: validez factorial del cuestionario de orientación emprendedora (COE). Revista Latinoamericana de Psicología. Vol. 42, $\mathrm{N}^{\circ}$ 1. España (Pp.41-52). Disponible en: http:/ / www.redalyc.org/articulo.oa?id=80515880004 consulta: 10/10/2019

Santamaría, Álvaro, Moreno, Eider, Torres, Eliany y Cadrazco, Wilson. (2013).La influencia de la familia en el emprendimiento empresarial: El caso de tres pymes de los sectores comercial y de servicios de la ciudad de Sincelejo Sucre. Revista Pensamiento Gerencial. No 1 . Bolivia. Recuperado de https://revistas.unisucre.edu.co/index.php/rpg/article/view/86 Consulta: 12/11/2019.

Sobrado, Luís y Fernández, Elena. (2010). Competencias emprendedorasy desarrollo del espíritu empresarial en los centros educativos. Revista Educación XX1. Vol. 13, No 1. España (Pp.15-38). DOI: https://doi.org/10.5944/educxx1.13.1.275

Toapanta, Gladys; Paucar, Amanda; Chango, Mercedes; Córdova, Alba; Guerrero, Mario y Cedeño, Dolores. (2018). El estudiante emprendedor en la universidad como importante factor de cambio en la sociedad. Revista Didasc@lia: Didáctica y Educación. Vol. 9, 
No1. Cuba (Pp.71-80). Disponible https://pdfs.semanticscholar.org/49eb/ba634ad305f143b52d2a79a3271de7fe7d08.pdf? $\mathrm{ga}=2.149579766 .1527320309 .1574542355-1607142921.1574542355 \quad$ consulta: $10 / 10 / 2019$

Whetten, David y Cameron, Kin. (2004). Desarrollo de habilidades directivas (sexta edición). Editorial Pearson Educación. México. 